\title{
CD99-positive soft tissue sarcoma with chromosomal translocation between 1 and 16 and inversion of chromosome 5
}

\author{
MASAHIKO KANAMORI ${ }^{1,2}$, KAYO SUZUKI ${ }^{2}$, TAKETOSHI YASUDA ${ }^{2}$ and TAKESHI HORI ${ }^{2}$ \\ Departments of ${ }^{1}$ Human Science 1, and ${ }^{2}$ Orthopaedics, University of Toyama, Toyama City, Toyama 930-0194, Japan
}

Received December 19, 2011; Accepted January 30, 2012

DOI: $10.3892 / \mathrm{ol} .2012 .641$

\begin{abstract}
In this study, we report the cytogenetic analysis of a 31-year-old male with a rare translocation between chromosomes 1 and 16 and inversion of chromosome 5 in CD99-positive soft tissue sarcoma of the thigh, which metastasized to the lung. Histologically, the tumor showed ovoid or short-spindle atypical cells with positivities of CD99, vimentin and bcl-2. Cytogenetically, all 20 analyzed cells showed the clonal aberrations add(1)(q23), $\mathrm{t}(1 ; 16)(\mathrm{p} 21 ; \mathrm{p} 11.2), \operatorname{inv}(5)$ (q11.2;q15). This finding adds to the new karyotype spectrum of CD99-positive soft tissue sarcomas.
\end{abstract}

\section{Introduction}

Certain types of soft tissue tumor are positive for CD99. CD99 is a membranous protein that is expressed in most cases of Ewing sarcoma (EWS), synovial sarcoma (SS) and low-grade fibromyxoid sarcoma (LGFMS), although its involvement in these diseases is unknown. However, knocking down CD99 expression in human EWS cell lines reduced their ability to form tumors and bone metastases when xenografted into immunodeficient mice, and diminished their tumorigenic characteristics in vitro (1). Therefore, CD99 may be a marker of aggressiveness. Some of these cancer types have a wellknown specific chromosomal translocation, such as $\mathrm{t}(11 ; 22)$ in EWS, or $\mathrm{t}(18 ; \mathrm{X})$ in SS $(2,3)$. However, this is the first case of CD99-positive soft tissue sarcoma with $\mathrm{t}(1 ; 16)$ and inv(5).

This study was approved by the local Ethics Committee of the University of Toyama. The patient was informed that his data would be submitted for publication, and gave consent.

\section{Case report}

The patient was a 31-year-old Japanese male with normal growth and development who presented with a mass located on the right thigh. He had noted a rapidly enlarging mass two

Correspondence to: Professor Masahiko Kanamori, Department of Human Science 1, University of Toyama, 2630 Sugitani, Toyama City, Toyama 930-0194, Japan

E-mail: kanamori@med.u-toyama.ac.jp

Key words: CD99, karyotype, chromosome, sarcoma months previously. On examination, there was a $15 \times 13 \mathrm{~cm}$ firm and non-tender tumor of the postero-medial thigh. The clinical history of the patient was normal.

Radiographs showed a non-mineralized soft tissue mass without bone involvement. T1- and T2-weighted MRI revealed a multi-lobulated heterogeneous large mass involving most of the flexor muscles, suggestive of a soft tissue sarcoma (Fig. 1). An angiogram revealed the hypervascularity of the tumor (Fig. 2). No other tumor or inguinal lymphadenopathy was present. Distal neurovascular examination was normal and laboratory findings were almost normal.

A needle biopsy of the tumor was carried out and the pathology was reviewed to be unclassified high-grade sarcoma (Fig. 3). The tumor was then surgically removed. However, the evaluation of the resected margin of the tumor was intralesional, since the mass had existed in the intermuscular space and lacked any capsule. Gross appearance of the resected specimen showed a non-capsulated, soft-to-firm, tan-to-pink mass (15 $\mathrm{cm}$ in maximum dimension) with foci of cysts and hemorrhage.

Microscopically, the tumor was composed of well-formed and variably sized vessels with proliferative ovoid or shortspindle atypical cells. Numerous mitotic figures were present. The tumor cells were immunoreactive to CD99, vimentin, and bcl-2. However, they had no reactivity to desmin, S-100, CD34, AE1/AE3, EMA and CK7.

Following surgery, the patient repeatedly received intensive multidrug chemotherapy, including combination chemotherapy VDC (vincristine $2 \mathrm{mg} /$ day, doxorubicin $50 \mathrm{mg} /$ day x 2 days, and cyclophosphamide $1800 \mathrm{mg} /$ day), IE (ifosfamide $3 \mathrm{~g}$ x 3 days, etoposide $100 \mathrm{mg} /$ day x 3 days) and MAID (doxorubicin $30 \mathrm{mg} / \mathrm{day}$, ifosfamide $3 \mathrm{~g} / \mathrm{day}$, dacarbazine $450 \mathrm{mg}$ /day) x 3 days (4). However, multiple lung metastatic tumors developed during postoperative chemotherapy. Moreover, the tumor recurred and metastasized to the retroperitoneal space at 2 years after surgery, and the patient suddenly succumbed to tumor-related septic shock 4 years after surgery.

Cytogenetic findings. Cytogenetic analysis was performed on G-band by trypsin and Giemsa (GTG) banding using surgical specimens. Standard culture and harvesting procedures were utilized, as described previously (5). The karyotypes were expressed according to the International System for Human Cytogenetic Nomenclature (6). Cells $(n=20)$ were analyzed. 

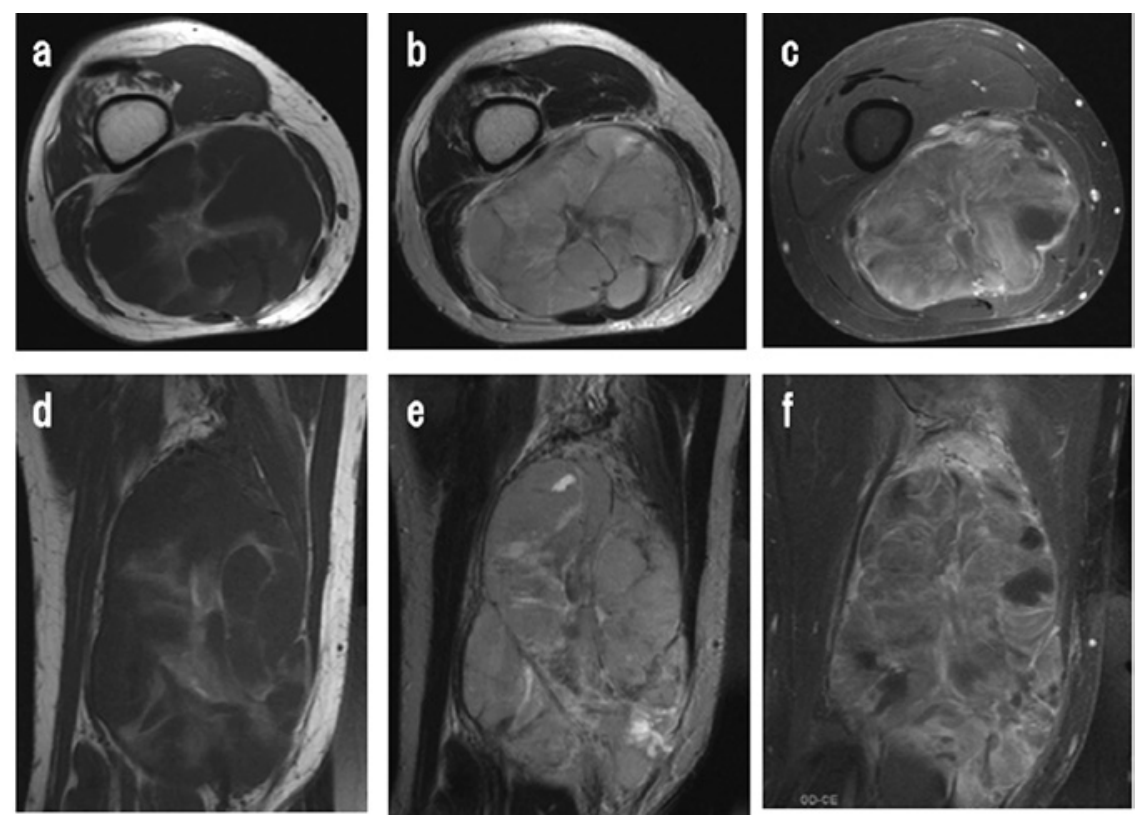

Figure 1. (a and d) Axial and sagittal T1-weighted (TR/TE; 640/20) spin echo images of the right thigh show a well-defined, heterogeneously hypointense mass. The mass showed the isointensity to the muscle with irregular septum. (b and e) Axial and sagittal T2-weighted (TR/TE; 180/3300) spin echo images show a multi-lobulated hyperintense mass involving most of the flexor muscles. (c and f) Gd-DTPA enhances the mass in a heterogeneous manner.

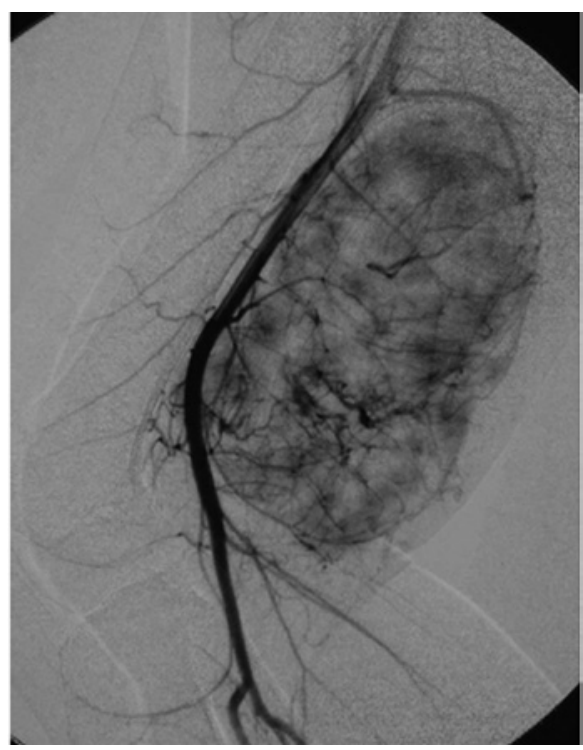

Figure 2. An angiogram shows the hypervascular tumor.

All 20 cells showed a clonal translocation involving chromosome 1 and 16, and inversion $5[46, \mathrm{XY}$, add(1)(q23), $\mathrm{t}(1 ; 16)$ (p21;p11.2), inv(5)(q11.2;q15)] (Fig. 4).

\section{Discussion}

Histopathologically, whereas the expression of CD99 is high in EWS (2) and in certain cases of SS, a low level of expression of CD99 is found in LGFMS, rhabdomyosarcoma, lymphomas, mesenchymal chondrosarcoma, osteosarcoma and neuroendocrine carcinoma $(1,7,8)$. CD99 may act as a tumor suppressor. Some of these tumors are difficult to diagnose differentially

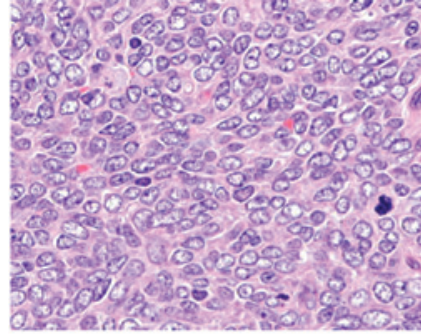

a

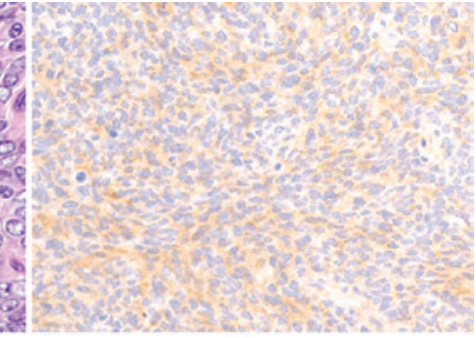

b
Figure 3. (a) Histopathology shows proliferative ovoid or short-spindle atypical cells. Numerous mitotic figures are present (H\&E; magnification, x20). (b) Immunohistochemistry shows the positivity for CD-99 (magnification, x200).

under microscopy, including immunohistochemical staining. Therefore, cytogenetic analysis is required for further tumor classification. For example, most EWS contain a pathognomonic translocation of $\mathrm{t}(11 ; 22)(\mathrm{q} 24 ; \mathrm{q} 12), \mathrm{t}(21 ; 22)(\mathrm{q} 22.2 ; \mathrm{q} 12)$ or $\mathrm{t}(7 ; 22)(\mathrm{p} 22 ; \mathrm{q} 12)$, fusing the EWS gene on chromosome 22 , which is a probable pivot event in the tumorigenesis of this neoplasm (9). In addition, most SS exhibit a well-known pathognomonic translocation of $\mathrm{t}(18 ; \mathrm{X})(\mathrm{p} 11 ; \mathrm{q} 11)$, and most LGFMS exhibit $\mathrm{t}(11 ; 16)(\mathrm{p} 11 ; \mathrm{p} 11)$.

Our case supports the possibility of a gene or genes at chromosomes 1, 5 and 16 . These genes may be important in tumorigenesis. Three components of cytogenetic aberration were observed in our case. The aberration add(1)(q23) has been non-specifically reported thus far, but neither $\mathrm{t}(1 ; 16)(\mathrm{p} 21 ; \mathrm{p} 11.2)$ nor $\operatorname{inv}(5)(\mathrm{q} 11.2 ; \mathrm{q} 15)$ were found in the NCBI database (http:// www.ncbi.nlm.njh.gov/sites/cancerchromosomes). To the best of our knowledge, this is the first case of sarcoma with $t(1 ; 16)$ (p21;p11.2) and inv(5)(q11.2;q15). 


\section{Acknowledgements}

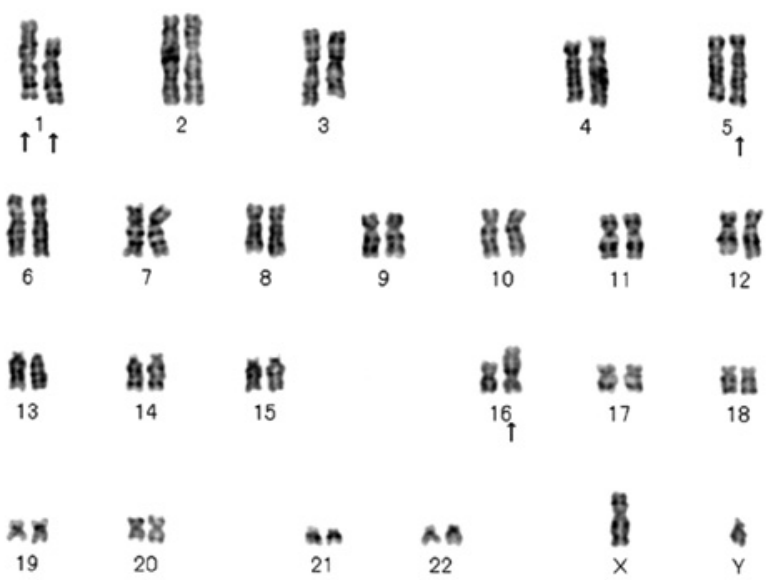

Figure 4. GTG banding analysis of all 20 metaphase chromosomes shows [46,XY, add(1)(q23), t(1;16)(p21;p11.2), inv(5)(q11.2;q15)].

FUS gene (also known as TLS) is encoded on chromosome band $16 \mathrm{p} 11$, and this location is specific for LGFMS. FUS gene transcription may now be used as a diagnostic marker for LGFMS. The FUS-CREB3L2 fusion gene transcripts were observed in $95 \%$ of well-defined LGFMS. In addition, the FUS-CREB3L1 variant resulting from $\mathrm{t}(11 ; 16)(\mathrm{p} 11 ; \mathrm{p} 11)$ was observed in $5 \%$. The two chimeric genes encode transcription activating proteins to form LGFMS (7). The immunohistochemical observations of CD99 positivity and CD34 negativity in LGFMS are similar to our case, but our case is high-grade, rather than low-grade sarcoma. Although 16 p11 in our case may be associated with FUS gene, we were unable to clarify the specific event that affects the tumorigenesis, as $\mathrm{t}(1 ; 16)(\mathrm{p} 21 ; \mathrm{p} 11.2)$ has yet to be reported (NCBI database). Moreover, $\operatorname{inv}(5)$ is also a novel constitutional aberration in soft tissue tumors.

The reduction of CD99 expression in human EWS cell lines results in neural differentiation, although its function in the disease is unknown. EWS revealed an inverse correlation between CD99 and neural markers, as well as an inverse correlation between neural differentiation and oncogenic transformation (1). Therefore, CD99 expression may correlate with tumorigenesis and malignant potential. Further investigation is required to elucidate CD99-positive tumors.
The authors would like to thank Professor Tomoatsu Kimura, of the Department of Orthopaedics, University of Toyama, who provided clinical advice.

\section{References}

1. Rocci A, Manara MC, Sciandra M, et al: CD99 inhibits neural differentiation of human Ewing sarcoma cells and thereby contributes to oncogenesis. J Clin Invest 120: 668-680, 2010.

2. Sandverg AA and Bridge JA: The Cytogenetics of Bone and Soft Tissue Tumors. RG Landes, Austin, pp288-312, 430-453, 1994.

3. Zucman J, Melot T, Desmaze C, et al: Combinational generation of variable fusion proteins in the Ewing family of tumors. EMBO J 12: 4481-4487, 1993.

4. Elias A, Ryan L, Sulkes A, et al: Response to mesna, doxorubicin, ifosfamide, and dacarbazine in 108 patients with metastatic or unresectable sarcoma and no prior chemotherapy. J Clin Oncol 7: 1208-1216, 1989

5. Bridge JA, Sreekantaiah C, Mouron B, et al: Clonal chromosomal abnormalities in desmoid tumors: implications for histopathogenesis. Cancer 69: 430-436, 1992.

6. ISCN. An International System for Human Cytogenetic Nomenclature. Mitelman F (ed), Karger, Basel, 1995.

7. Guillou L, Benhattar J, Gengler C, et al: Translocationpositive low grade fibromyxoid sarcoma: clinicopathological and molecular analysis of a series expanding the morphologic spectrum and suggesting potential relationship to sclerosing epithelioid fibrosarcoma: a study from the French Sarcoma Group. Am J Surg Pathol 31: 1387-1402, 2007.

8. Malone VS, Dobin SM, Jones KA, et al: CD99-positive large cell neuroendocrine carcinoma with rearranged EWSR1 gene in an infant: a case of prognostically favorable tumor. Virchows Arch 457: 389-395, 2010.

9. Desmaze C, Brizard F, Turc-Carel C, et al: Multiple chromosomal mechanisms generate an EWS/ FLI1 or an EWS/ ERG fusion gene in Ewing tumors. Cancer Genet Cytogenet 97: 12-19, 1997. 